\title{
Kimura's Disease: A Case Report in a Child
}

\author{
T. Shankar $\cdot$ Neeraja MyReddy $\cdot$ K. P. Varalaxmi
}

Received: 26 July 2010/ Accepted: 26 December 2011/Published online: 11 February 2012

(C) Association of Otolaryngologists of India 2012

\begin{abstract}
Kimura's disease is chronic inflammatory disorder of unknown etiology that most commonly present as painless, unilateral cervical lymphadenopathy, males are more commonly effected than females with 3.5:1-9:1 ratio. Kimura's disease commonly seen in young adults median age being 28-32 years. Here we are presenting a case of Kimura's disease in a 6 year old male child, with a bilateral presentation. The goals of pharmacotherapy for Kimura's disease are to reduce morbidity and to prevent complications. The prognosis for Kimura's disease is good with no potential for malignant transformation.
\end{abstract}

Keywords Kimura's disease - Eosinophilic lymphogranuloma · Cervical lymphadenopathy · Salivary gland hypertrophy $\cdot$ Nephrotic syndrome

\section{Introduction}

- Kimura's disease is a chronic inflammatory disorder of unknown etiology that most commonly presents as painless, unilateral cervical lymphadenopathy or subcutaneous masses in the head and neck region.

- The first report of Kimura's disease was from China in 1937.

- The disorder received its current name in 1948, when Kimura's et al. [2] noted the vascular component and

T. Shankar $(\bowtie)$

Department of ENT, Government Medical College, Anantapur,

Andhra Pradesh, India

e-mail: drshankar_ms@yahoo.com

N. MyReddy · K. P. Varalaxmi

Department of Pathology, Government Medical College,

Anantapur, Andhra Pradesh, India referred to it as "unusual granulation combined with hyperplastic changes in lymphoid tissue."

- The Kimura's disease may represent a primary inflammatory process with secondary vascular proliferation.

\section{Case Report}

A 6 year old boy by name J. Naveen Kumar of Anantapur district, Andhra Pradesh was brought to ENT Department OPD (O.P.No.1787) with complaints of bilateral post aural swelling since 3 years, no other complaints were present.

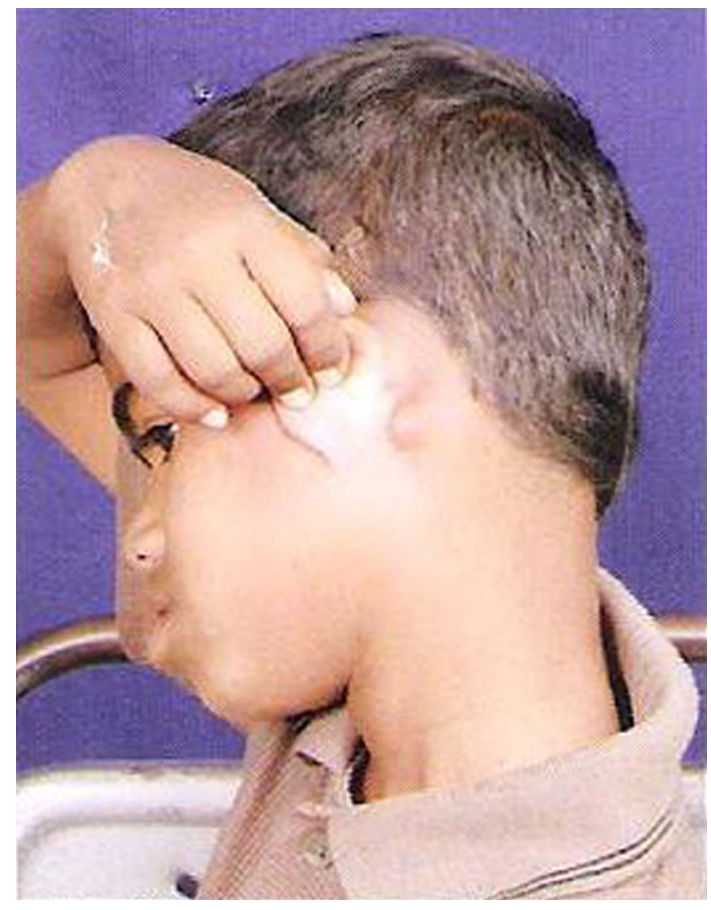

On examination small, single round swelling bilaterally mobile freely (size $1 \mathrm{~cm} \times 0.5 \mathrm{~cm}$ ) present post aurally. 
Patient was investigated routinely all the biochemical investigations found to be normal except $\uparrow$ eosinophils, $\uparrow$ serum IgE levels and patient was advised for FNAC. The FNAC was done (FN/95/10) shows moderate cellularity consisting of population of lymphocytes, immunoblasts and good number of eosinophils with these features possibility of Kimura's disease is considered and advised for Biopsy for confirmation. Subsequently patient was admitted (IP.No.9738) in ENT Department posted for excision biopsy, biopsy was done $\downarrow$ G.A and sent for HPE. The patient was discharged on 3rd day, suture removal was done on 7th day the histopathology report came on 28-4-2010 (Biopsy No.B/105/10) confirms Kimura's disease.

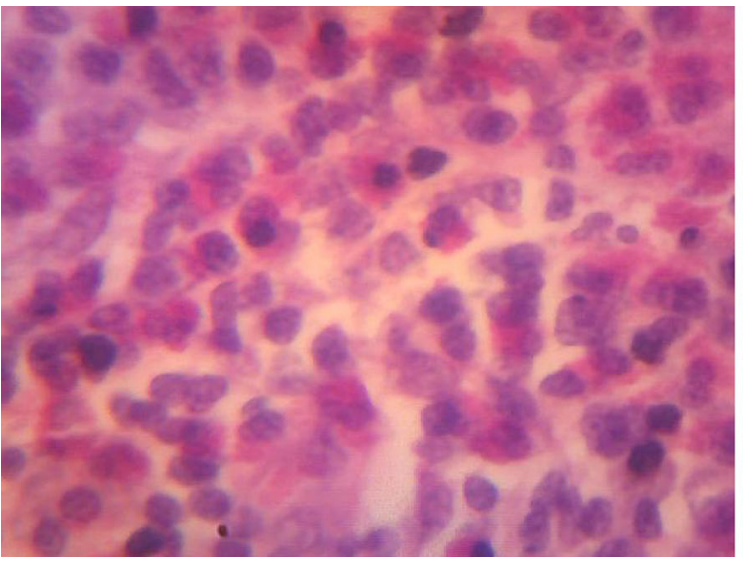

Sections studied show, thickened capsule with cortical, follicular hyperplasia. Proliferation of capillaries with prominent endothelial cells. There are abundant numbers of eosinophilis in the Para cortical region. Peri nodal adipose tissue is infiltrated by few lymphocytes. Few areas show extensive fibrosis around the blood vessels. Histological features are suggestive of Kimura's disease (Angiofollicular hyperplasia with eosinophilic infiltration.

As the patient is child the case is also referred to pediatric consultation and kept on Tab. Prednisone $20 \mathrm{mg} /$ day Taper to $5 \mathrm{mg} /$ week for 6 week. Patient is under follow up no further complication noted.

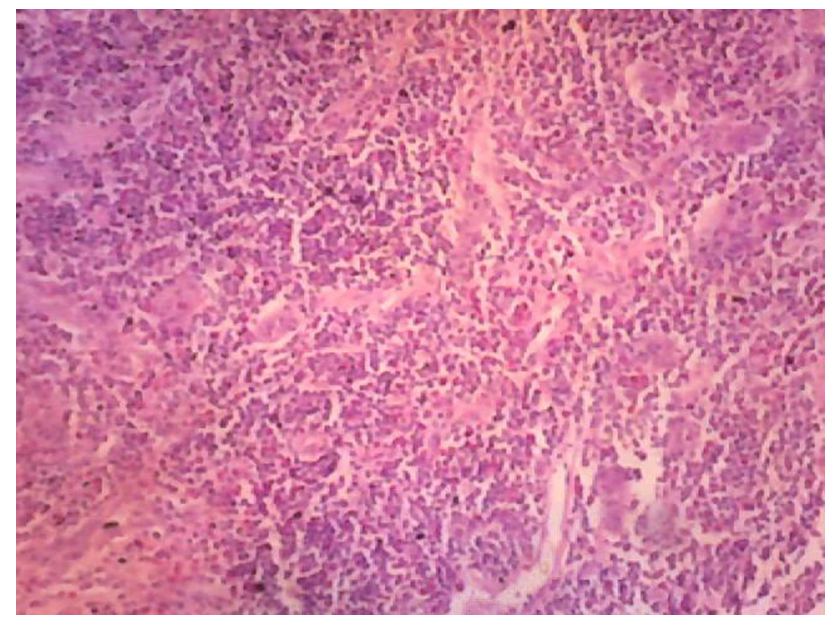

\section{Discussion}

Kimura's disease is a chronic inflammatory disorder of unknown etiology that most commonly presents as painless, unilateral cervical lymphadenopathy or subcutaneous masses in the head or neck region. The first report of Kimura's disease was from China in 1937, in which Kimm and Szeto [1] descirbed 7 cases of a condition they termed "eosinophilic hyperplastic lymphogranuloma.: The disorder received its current name in 1948, when Kimura's et al. [2] noted the vascular component and referred to it as an "unusual granulation combined with hyperplastic

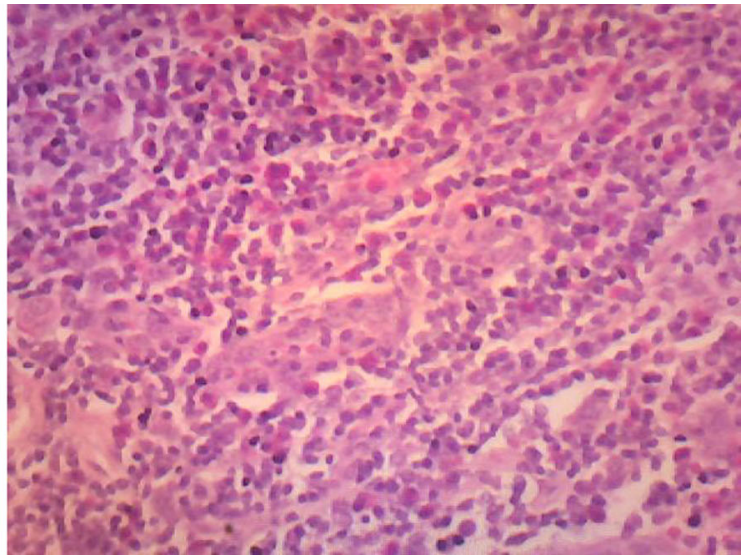

changes in lymphoid tissue." Controversy exists in the literature regarding whether Kimura's disease and angiolymphoid hyperplasia with eosinophilia (ALHE) are the same entity. Some authors believe that Kimura's disease represents a chronic, defer from of ALHE; however, most recent papers distinguish the two on the basis of clinical and histopathologic characteristics. ALHE appears to represent an arterivenous malformation and secondary inflammation. Kimura's disease may represent a primary inflammatory process with secondary vascular proliferation.

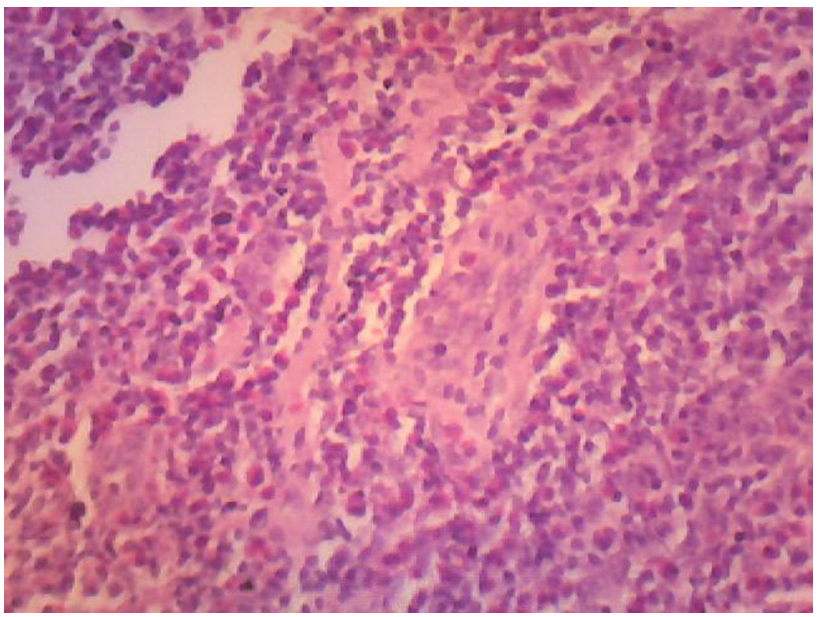


The patholophysiology of Kimura's disease remains unknown, although an allergic reaction, trauma, and an autoimmune process have been implicated as possible causes. Kimura's disease is manifested by an abnormal proliferation of lymphoid follicles and vascular endothelium. Peripheral eosinophilia and the presence of eosinophils in the inflammatory infiltrate suggest that Kimura's disease may be a hypersensitivity reaction. Some evidence has indicated that the interaction between $T_{H} 1$ and $T_{H} 2$ lymhocytes may result in the excessive production of eosinophilotrophic cytokines, such as interleukin 4. Persistent antigenic stimulation from insect bites, parasitic infestation, candidal infection, or viral infection may cause the activation of this cytokine pathway, but further investigation is needed [3, 4]. Kimura's disease involves the skin, lymph nodes, and salivary glands and is reported to be associated with nephrotic syndrome in approximately $15-19 \%$ of cases. The basis of this possible association is not well understood $[5,6]$.

Kimura's disease has rarely been reported in the United States. The exact prevalence of Kimura's disease is not known. Most cases of this rare disease are reported in East and Southeast Asia, with a small number of cases reported in Europe [7]. Kimura's disease is a benign disorder with no potential for malignant transformation, but spontaneous involution is rare. The main concern is the capacity for lesions to grow and cause disfigurement. Recurrence have been reported after medical and surgical treatment of Kimura's disease. Males are affected by Kimura's disease more commonly than females, with a 3.5:1-9:1 male-to-female ratio is most series reported, with the exception of one series in which the male-to-female ratio was 19:1 [6]. Kimura's disease is usually seen in young adults during the third decade of life, with the median age being 28-32 years [3, 8, 9].

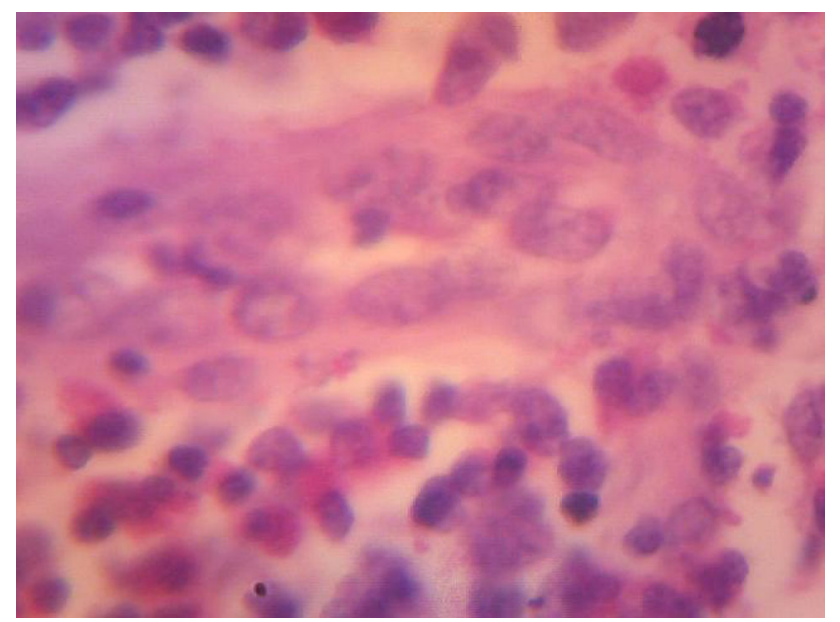

The Lesions of Kimura's disease typically are slowgrowing, painless masses with occasional pruritus of the overlying skin. Patients with Kimura's disease may present with a solitary enlarged painless lymph node or generalized lymphadenopathy. Salivary gland involvement is also frequently observed. Other findings include single or multiple pink-to-red cutaneous nodules, which are usually located on the head or neck, especially in the periauricular, parotid, or submandibular regions. Less frequently, the eyelids, orbit, and lacrimal glands may be involved. The average diameter of lesions is $3 \mathrm{~cm}$. Although Kimura's disease mainly affects the head and neck, involvement of the extremities and inguinal lymph nodes has been reported. The exact etiology of Kimura's disease is unknown, but it has been proposed that the interaction between $\mathrm{T}_{\mathrm{H}} 1$ and $\mathrm{T}_{\mathrm{H}} 2$ lymphocytes results in an abnormal production of eosinophils and immunoglobulin E. an inciting autoimmunity, allergic reaction or an alteration of immune regulation is suspected. Proposed theories include persistent antigenic stimulation following arthropod bites, parasitic infestation, or viral or candidal infection. However, none of these theories has been substantiated.

Laboratory Investigations:

- CBP count with differential almost always reveals peripheral eosinophilia is persons with Kimura's disease $(98 \%)$. The number of eosinophils was closely correlated to the sizes of the neck masses in one series [10].

- Serum immunoglobulin E levels are often elevated in person with Kimura's disease.

- Blood urea nitrogen, creatinine, and urinary protein levels should be obtained to exclude concomitant renal dysfunction and/or nephrotic syndrome.

- Serum eosinophil cationic protein levels parallel the course of the disease [11].

- Ultrasonography, CT scanning, or MRI can help determine the extent of the disease. MRI and ultrasonography produce characteristic findings, but results from CT examination have been nonspecific for Kimura's disease [12].

- Incisional biopsy is recommend to obtain the diagnosis of Kimura's disease.

\section{Histological Findings}

Lymphoid nodules with discrete germinal centres can occupy an area extending from the reticular dermis to the fascia and muscle. A marked eosinophilic infiltrate and eosinophilic abscesses are present. Centrally, thick-walled vessels are present with hobnail endothelial cell. Immunohistochemical evaluation of the lymphoid nodules demonstrates a polymorphous infiltrate without clonality. 


\section{Treatment}

\section{Medical Care}

- Observation is acceptable if the Kimura's disease lesions are neither symptomatic nor disfiguring.

- Intralesional or oral steroids can shrink the nodules but seldom result in cure. A medium-potency steroid (e.g. Triamcinolone acetonide), used in solution form for intralesional injection, is usually well tolerated.

- Radiotherapy has occasionally been used to treat recurrent or persistent Kimura's disease lesions. A report by Hareyama et al. [13] reported on the use of radiotherapy at dosages of 26-30 Gy; local control was achieved in $74 \%$ of lesions. Another study demonstrated that radiotherapy (20-45 Gy) was more effective than local excision and steroid treatment, with local response rates of $64.3 \%$ versus $22.2 \%$, respectively. However, considering the being nature of Kimura's disease, further investigation may be required, and caution using radiation outside of recurrent, disfiguring lesions is required.

- Cyclosporine has been reported to induce remission in patients with Kimura's disease. A dose of $5 \mathrm{mg} / \mathrm{kg} / \mathrm{d}$ was effective, but, in most cases, the lesions recurred upon cessation of therapy [14].

- Oral pentoxifylline has been reported to be effective in one patient with Kimura's disease; however, the lesions relapsed after discontinuation of therapy [15].

- All trans-retinoic acid in combination of prednisone has resulted in remission of Kimura's disease in one patient, and he remained disease free 12 months after discontinuation of all therapy [16].

- Imatinib may be effective treatment for Kimura's disease, based on advances in research for therapy in hypereosinophilic syndrome, but further investigation is necessary [7].

\section{Surgical Care}

Conservative surgical excision is considered the treatment of choice for Kimura's disease; however, lesions often recur after excision. Consultation with an ophthalmologist and Otolaryngologist should be considered for further evaluation depending on the extent and location of the disease. Rarely large nodules or tumors from Kimura's disease have ulcerated. After excision, recurrence also has been reported.

The prognosis for Kimura's disease is good, with no potential for malignant transformation. The course of Kimura's disease is chronic, with lesions frequently persisting or recurring despite treatment. Failure to recognize and treat Kimura's disease may result in the development of large and disfiguring lesions.

\section{Conclusion}

Kimura's disease is a chronic inflammatory disorder of unknown origin that most commonly present as painless unilateral cervical lymphadenopathy, the pathophysiology of Kimura's disease remains un-known, although an allergic reaction, trauma and an auto-immun process have also been implicated as possible causes, males are effected by Kimura's disease more commonly than females in age group of 28-32, but in our case the age is about 6 years of male child, the prognosis of Kimura's disease is good with no potentially for malignant transformation.

\section{References}

1. Kimm HT, Szeto C (1937) Eosinophilic hyperplastic lymphogranuloma, comparison with Mikulicz's disease. Proc Chin Med Soc 329

2. Kimura's T, Yoshimura S, Ishikawa E (1948) On the unusual granulation combined with hyperplastic changes of lymphatic tissues. Trans Soc Pathol Jpn 37:179-180

3. Thomas J, Jayachandran NV, Chandrasekhara PK, Rajasekhar L, Narsimulu G (2008) Kimura's disease-an unusual cause of lymphadenopathy in children. Clin Rheumatol 27(5):675-677

4. Mrówka-Kata K, Kata D, Kyrcz-Krzemien S, Helbig G (2009) Kikuchi-Fujimoto and Kimura's diseases: the selected, rare causes of neck lymphadenopathy. Eur Arch Otorhinolaryngol Oct 16 2009

5. Rajpoot DK, Pahl M, Clark J (2000) Nephrotic syndrome associated with Kimura's disease. Pediatr Nephrol 14(6):486-488

6. Wang DY, Mao JH, Zhang Y et al (2009) Kimura's disease: a case report and review of the Chinese literature. Nephron Clin Pract 111(1):c55-c61

7. Sun QF, Xu DZ, Pan SH et al (2008) Kimura's disease: review of the literature. Intern Med J 38(8):668-672

8. Kung IT, Gibson JB, Bannatyne PM (1984) Kimura's disease: a clinico-pathological study of 21 cases and its distinction from angiolymphoid hyperplasia with eosinophilia. Pathology 16(1):39-44

9. Chen H, Thompson LD, Aguilera NS, Abbondanzo SL (2004) Kimura's disease: a clinicopathologic study of 21 cases. Am J Surg Pathol 28(4):505-513

10. Masayuki S, Ayako K, Shinichi N (2005) Hematoserological analysis of Kimura's disease for optimal treatment. Otolaryngol Head Neck Surg 132:159-160

11. Ohta N, Okazaki S, Fukase S, Akatsuka N, Aoyagi M, Yamakawa M (2007) Serum concentrations of eosinophil cationic protein and eosinophils of patients with Kimura's disease. Allergol Int 56(1):45-49

12. Takeishi M, Makino Y, Nishioka H, Miyawaki T, Kurihara K (2007) Kimura's disease: diagnostic imaging findings and surgical treatment. J Craniofac Surg 18(5):1062-1067 
13. Hareyama M, Oouchi A, Nagakura H et al (1998) Radiotherapy for Kimura's disease: the optimum dosage. Int $\mathrm{J}$ Radiat Oncol Biol Phys 40(3):647-651

14. Kaneko K, Aoki M, Hattori S, Sato M, Kawana S (1999) Successful treatment of Kimura's disease with cyclosporine. J Am Acad Dermatol 41(5 Pt 2):893-894
15. Hongcharu W, Baldassano M, Taylor CR (2000) Kimura's disease with oral ulcers: response to pentoxifylline. J Am Acad Dermatol 43(5 Pt 2):905-907

16. Boulanger E, Gachot B, Verkarre V, Valensi F, Brousse N, Hermine O (2002) all-trans-Retinoic acid in the treatment of Kimura's disease. Am J Hematol 71(1):66 